\title{
NORMAL CYCLIC POLYTOPES AND CYCLIC POLYTOPES THAT ARE NOT VERY AMPLE
}

\author{
TAKAYUKI HIBI, AKIHIRO HIGASHITANI ${ }^{凶}$, LUKAS KATTHÄN \\ and RYOTA OKAZAKI
}

(Received 3 December 2012; accepted 8 July 2013; first published online 30 September 2013)

\author{
Communicated by G. Willis
}

\begin{abstract}
Let $d$ and $n$ be positive integers such that $n \geq d+1$ and $\tau_{1}, \ldots, \tau_{n}$ integers such that $\tau_{1}<\cdots<\tau_{n}$. Let $C_{d}\left(\tau_{1}, \ldots, \tau_{n}\right) \subset \mathbb{R}^{d}$ denote the cyclic polytope of dimension $d$ with $n$ vertices $\left(\tau_{1}, \tau_{1}^{2}, \ldots, \tau_{1}^{d}\right)$, $\ldots,\left(\tau_{n}, \tau_{n}^{2}, \ldots, \tau_{n}^{d}\right)$. We are interested in finding the smallest integer $\gamma_{d}$ such that if $\tau_{i+1}-\tau_{i} \geq \gamma_{d}$ for $1 \leq i<n$, then $C_{d}\left(\tau_{1}, \ldots, \tau_{n}\right)$ is normal. One of the known results is $\gamma_{d} \leq d(d+1)$. In the present paper a new inequality $\gamma_{d} \leq d^{2}-1$ is proved. Moreover, it is shown that if $d \geq 4$ with $\tau_{3}-\tau_{2}=1$, then $C_{d}\left(\tau_{1}, \ldots, \tau_{n}\right)$ is not very ample.
\end{abstract}

2010 Mathematics subject classification: primary 52B20; secondary 05E40, 05E45.

Keywords and phrases: cyclic polytope, normal polytope, very ample polytope.

\section{Introduction}

The cyclic polytope is one of the most distinguished polytopes and played the essential role in the classical theory of convex polytopes [2]. Let $d$ and $n$ be positive integers such that $n \geq d+1$ and $\tau_{1}, \ldots, \tau_{n}$ real numbers such that $\tau_{1}<\cdots<\tau_{n}$. The convex polytope $C_{d}\left(\tau_{1}, \ldots, \tau_{n}\right)$ which is the convex hull of the finite set

$$
\left\{\left(\tau_{1}, \tau_{1}^{2}, \ldots, \tau_{1}^{d}\right), \ldots,\left(\tau_{n}, \tau_{n}^{2}, \ldots, \tau_{n}^{d}\right)\right\} \subset \mathbb{R}^{d}
$$

is called a cyclic polytope. It is known that $C_{d}\left(\tau_{1}, \ldots, \tau_{n}\right)$ is a simplicial polytope of dimension $d$ with $n$ vertices. The combinatorial type of $C_{d}\left(\tau_{1}, \ldots, \tau_{n}\right)$ is independent of the particular choice of real numbers $\tau_{1}, \ldots, \tau_{n}$.

The first and fourth authors are supported by the JST CREST 'Harmony of Gröbner Bases and the Modern Industrial Society'. The second author is supported by the JSPS Research Fellowship for Young Scientists. This research was carried out while the third author was staying at Department of Pure and Applied Mathematics, Osaka University, between November 2011 and April 2012, supported by the DAAD.

(C) 2013 Australian Mathematical Publishing Association Inc. 1446-7887/2013 \$16.00 
The present paper is devoted to the study on integral cyclic polytopes. A convex polytope is called integral if all of its vertices have integer coordinates. The integral convex polytope has established an active area of research between combinatorics and commutative algebra [4, 9].

Let, in general, $\mathcal{P} \subset \mathbb{R}^{N}$ be an integral convex polytope, define $\mathcal{P}^{*} \subset \mathbb{R}^{N+1}$ to be the convex hull of all points $(1, \alpha) \in \mathbb{R}^{N+1}$ with $\alpha \in \mathcal{P}$ and let $\mathcal{A l}_{\mathcal{P}}=\mathcal{P}^{*} \cap \mathbb{Z}^{N+1}$ denote the set of integer points in $\mathcal{P}^{*}$. Let $\mathbb{Z}_{\geq 0}$ denote the set of nonnegative integers and $\mathbb{Q}_{\geq 0}$ the set of nonnegative rational numbers.

We say that $\mathcal{P}$ is normal if

$$
\mathbb{Z}_{\geq 0} \mathcal{A}_{\mathcal{P}}=\mathbb{Z}_{\mathcal{P}} \cap \mathbb{Q}_{\geq 0} \mathcal{A}_{\mathcal{P}}
$$

Moreover, $\mathcal{P}$ is called very ample if the set

$$
\left(\mathbb{Z}_{\mathcal{P}} \cap \mathbb{Q}_{\geq 0} \mathcal{A}_{\mathcal{P}}\right) \backslash \mathbb{Z}_{\geq 0} \mathcal{A}_{\mathcal{P}}
$$

is finite. One of the most fundamental questions on integral convex polytopes is to determine whether a given integral convex polytope is normal [6].

On the other hand, we say that an integral convex polytope $\mathcal{P} \subset \mathbb{R}^{N}$ has the integer decomposition property if, for each $m=1,2, \ldots$ and for each $\alpha \in m \mathcal{P} \cap \mathbb{Z}^{N}$, there exist $\alpha_{1}, \ldots, \alpha_{m}$ belonging to $\mathcal{P} \cap \mathbb{Z}^{N}$ such that $\alpha=\alpha_{1}+\cdots+\alpha_{m}$. Here $m \mathcal{P}=\{m \alpha: \alpha \in \mathcal{P}\}$. If $\mathcal{P}$ has the integer decomposition property, then $\mathcal{P}$ is normal. However, the converse is false. For example, the tetrahedron $\mathcal{T}_{3} \subset \mathbb{R}^{3}$ with the vertices $(0,0,0),(1,1,0),(1,0,1)$ and $(0,1,1)$ is normal, but cannot have the integer decomposition property because $(1,1,1) \in 2 \mathcal{T}_{3}$. If $\mathcal{P} \subset \mathbb{R}^{d}$ is an integral convex polytope of dimension $d$ with $\mathbb{Z}\left(\mathscr{P}^{*} \cap \mathbb{Z}^{d+1}\right)=\mathbb{Z}^{d+1}$, then $\mathcal{P}$ has the integer decomposition property if and only if $\mathcal{P}$ is normal. Lemma 2.6 says that every integral cyclic polytope $\mathcal{P} \subset \mathbb{R}^{d}$ satisfies $\mathbb{Z}\left(\mathcal{P}^{*} \cap \mathbb{Z}^{d+1}\right)=\mathbb{Z}^{d+1}$. In particular, it follows that an integral cyclic polytope is normal if and only if it has the integer decomposition property.

Let, as before, $d$ and $n$ be positive integers such that $n \geq d+1$. Given integers $\tau_{1}, \ldots, \tau_{n}$ with $\tau_{1}<\cdots<\tau_{n}$, we wish to examine whether $C_{d}\left(\tau_{1}, \ldots, \tau_{n}\right)$ is normal or not. Thus our final goal is to classify the integers $\tau_{1}, \ldots, \tau_{n}$ with $\tau_{1}<\cdots<\tau_{n}$ for which $C_{d}\left(\tau_{1}, \ldots, \tau_{n}\right)$ is normal. Even though it seems to be rather difficult to find a complete classification, many fascinating problems naturally arise. As a first step toward our goal, we are interested in finding the smallest integer $\gamma_{d}$ such that if $\tau_{i+1}-\tau_{i} \geq \gamma_{d}$ for $1 \leq i<n$, then $C_{d}\left(\tau_{1}, \ldots, \tau_{n}\right)$ is normal. Since the lattice length of each edge $\operatorname{conv}\left(\left\{\left(\tau_{i}, \ldots, \tau_{i}^{d}\right),\left(\tau_{j}, \ldots, \tau_{j}^{d}\right)\right\}\right)$ of $C_{d}\left(\tau_{1}, \ldots, \tau_{n}\right)$ coincides with $\left|\tau_{j}-\tau_{i}\right|$, it follows immediately from [3, Theorem 1.3(b)] that $\gamma_{d} \leq d(d+1)$. In the present paper a new inequality $\gamma_{d} \leq d^{2}-1$ is proved (Theorem 3.1). Moreover, it is shown that if $d \geq 4$ with $\tau_{3}-\tau_{2}=1$, then $C_{d}\left(\tau_{1}, \ldots, \tau_{n}\right)$ is not very ample (Theorem 4.1).

A brief overview of the present paper is as follows. After preparing notation, terminologies together with several lemmata in Section 2, a proof of Theorem 3.1 is achieved in Section 3. Section 4 is devoted to showing Theorem 4.1. An algebraic 
aspect of integral cyclic polytopes including toric rings of integral cyclic polytopes will be studied in the forthcoming paper [5].

\section{Preliminaries}

In this section, we prepare notation and lemmas for our main theorem.

First of all, we review some fundamental facts on cyclic polytopes. Let $d$ and $n$ be positive integers such that $n \geq d+1$. It is convenient to work with a homogeneous version of the cyclic polytopes, hence, throughout the present paper, we consider $C_{d}^{*}\left(\tau_{1}, \ldots, \tau_{n}\right)$ instead of $C_{d}\left(\tau_{1}, \ldots, \tau_{n}\right)$. For $n$ real numbers $\tau_{1}, \ldots, \tau_{n}$ with $\tau_{1}<$ $\cdots<\tau_{n}$, we set

$$
v_{i}:=\left(1, \tau_{i}, \tau_{i}^{2}, \ldots, \tau_{i}^{d}\right) \in \mathbb{R}^{d+1} \quad \text { for } 1 \leq i \leq n .
$$

In other words, $C_{d}^{*}\left(\tau_{1}, \ldots, \tau_{n}\right)=\operatorname{conv}\left(\left\{v_{i}: 1 \leq i \leq n\right\}\right) \subset \mathbb{R}^{d+1}$. Unless stated otherwise, we will always assume that the indices are ordered as $\tau_{1}<\cdots<\tau_{n}$. See [10, Ch. 0] for some basic properties of cyclic polytopes. We will use a well-known characterization of their facets. (See, for example, [10, Theorem 0.7].)

Let $[n]:=\{1, \ldots, n\}$ and let us say that a set $S \subset[n]$ forms a facet of $C_{d}^{*}\left(\tau_{1}, \ldots, \tau_{n}\right)$ if $\operatorname{conv}\left(\left\{v_{i}: i \in S\right\}\right)$ is its facet.

Proposition 2.1 (Gale's evenness condition). A set $S \subset[n]$ with $d$ elements forms a facet of $C_{d}^{*}\left(\tau_{1}, \ldots, \tau_{n}\right)$ if and only if $S$ satisfies the following condition: if $i$ and $j$ with $i<j$ are not in $S$, then the number of elements of $S$ between $i$ and $j$ is even. In other words,

$$
2 \mid \#\{k \in S \mid i<k<j\},
$$

where $\# X$ stands for the number of elements contained in a finite set $X$.

Henceforth, we will assume that $\tau_{1}, \ldots, \tau_{n}$ are integers.

Let $\Delta_{i j}:=\tau_{j}-\tau_{i}$ for $i, j \in[n]$. The proof of Proposition 2.1 yields a description of the inequality of the supporting hyperplane defining each facet. Let $S=\left\{k_{1}, \ldots, k_{d}\right\} \subset$ $[n]$ and consider the polynomial

$$
\sum_{i=0}^{d} c_{S, i} t^{i}:=\prod_{i \in S}\left(t-\tau_{i}\right)
$$

Then all $d$ vectors $v_{k_{1}}, \ldots, v_{k_{d}}$ vanish by the linear form

$$
\sigma_{S}: \mathbb{R}^{d+1} \ni\left(w_{0}, w_{1}, \ldots, w_{d}\right) \mapsto \sum_{i=0}^{d} c_{S, i} w_{i} \in \mathbb{R},
$$

thus it defines the hyperplane spanned by them. Note that we index the first coordinate by 0 . Hence, if the set $S$ forms a facet $\mathcal{F}$ of $\mathcal{P}^{*}=C_{d}^{*}\left(\tau_{1}, \ldots, \tau_{n}\right)$, then $\sigma_{S}$ is the linear form defining $\mathcal{F}$, which means that $\sigma_{S}(x) \geq 0$ if $x$ is in $\mathcal{P}^{*}$ and $\sigma_{S}(x)=0$ if $x$ is in $\mathcal{F}$. 
For every $j \in[n] \backslash S$, we have $\sigma_{S}\left(v_{j}\right)=\prod_{i \in S} \Delta_{i j}$. This has a useful implication, that is, if we write a vector $x \in \mathbb{Z}^{d+1}$ as $x=\sum_{i \in S} \lambda_{i} v_{i}+\lambda_{j} v_{j}$ with rational coefficients $\lambda_{i}$, then the denominator of $\lambda_{j}$ is a divisor of $\prod_{i \in S} \Delta_{i j}$, because $\sigma_{S}(x)=\lambda_{j} \prod_{i \in S} \Delta_{i j}$ is an integer.

We introduce a special representation of cyclic polytopes which is sometimes helpful. Write the vectors $v_{1}, \ldots, v_{n}$ as row vectors into a matrix, namely,

$$
\left(\begin{array}{c}
v_{1} \\
v_{2} \\
\vdots \\
v_{n}
\end{array}\right)=\left(\begin{array}{ccccc}
1 & \tau_{1} & \tau_{1}^{2} & \ldots & \tau_{1}^{d} \\
1 & \tau_{2} & \tau_{2}^{2} & \ldots & \tau_{2}^{d} \\
\vdots & \vdots & & & \vdots \\
1 & \tau_{n} & \tau_{n}^{2} & \ldots & \tau_{n}^{d}
\end{array}\right) .
$$

LEMMA 2.2. The aforementioned matrix can be transformed to the following matrix by using a unimodular transformation:

$$
\left(\begin{array}{ccccc}
1 & 0 & \cdots & \cdots & 0 \\
1 & \Delta_{12} & 0 & \ddots & \vdots \\
1 & \Delta_{13} & \Delta_{13} \Delta_{23} & \ddots & \vdots \\
\vdots & \vdots & \vdots & \ddots & 0 \\
1 & \Delta_{1, d+1} & \Delta_{1, d+1} \Delta_{2, d+1} & \ldots & \prod_{k=1}^{d} \Delta_{k, d+1} \\
\vdots & \vdots & \vdots & & \vdots \\
1 & \Delta_{1, n} & \Delta_{1, n} \Delta_{2, n} & \ldots & \prod_{k=1}^{d} \Delta_{k, n}
\end{array}\right) .
$$

In particular, the convex hull of the row vectors of this matrix is unimodularly equivalent to $C_{d}^{*}\left(\tau_{1}, \ldots, \tau_{n}\right)$.

A proof of the above lemma is essentially the same as a proof of the well-known Vandermonde determinant identity. Note that Lemma 2.2 is valid for any ordering of the parameters $\tau_{1}, \ldots, \tau_{n}$, that is, any ordering of $v_{1}, \ldots, v_{n}$.

Let us identify a special case where the polytopes are indeed unimodularly equivalent.

Lemma 2.3. An integral cyclic polytope $C_{d}^{*}\left(\tau_{1}, \ldots, \tau_{d}\right)$ is unimodularly equivalent to $C_{d}^{*}\left(-\tau_{n}, \ldots,-\tau_{1}\right)$. Moreover, for any integer $m, C_{d}^{*}\left(\tau_{1}, \ldots, \tau_{d}\right)$ is unimodularly equivalent to $C_{d}^{*}\left(\tau_{1}+m, \ldots, \tau_{n}+m\right)$.

Proof. The replacement $\tau_{i} \mapsto-\tau_{i}$ corresponds to a multiplication with -1 in every column of (2.1) with an odd exponent. This is a unimodular transformation. The second statement is immediate from Lemma 2.2, because the matrix (2.2) depends only on the differences $\Delta_{i j}=\tau_{j}-\tau_{i}$. 
We define a certain class of vectors which we will use in what follows. Let $S=\left\{i_{1}, \ldots, i_{q}\right\} \subset[n]$ be a nonempty set, where $i_{1}<\cdots<i_{q}$. Then we define

$$
b_{S}:=\sum_{i \in S} \frac{1}{\prod_{j \in S \backslash\{i\}} \Delta_{i j}} v_{i}=\sum_{k=1}^{q} \frac{(-1)^{k+1}}{\prod_{j \in S \backslash\left\{i_{k}\right\}}\left|\Delta_{i_{k} j}\right|} v_{i_{k}},
$$

where $b_{S}=v_{i_{1}}$ when $q=1$, that is, $\# S=1$. If $S$ is small, we will sometimes omit the brackets around the elements, thus we write, for example, $b_{i j}=b_{\{i, j\}}$. However, the vector does not depend on the order of the indices.

ExAmple 2.4. Let us write down $b_{S}$ for small sets $S$. Assume that $1 \leq i<j<k<l \leq n$. Then

$$
\begin{aligned}
b_{i} & =v_{i}, \\
b_{i j} & =\frac{1}{\Delta_{i j}} v_{i}-\frac{1}{\Delta_{i j}} v_{j}, \\
b_{i j k} & =\frac{1}{\Delta_{i j} \Delta_{i k}} v_{i}-\frac{1}{\Delta_{i j} \Delta_{j k}} v_{j}+\frac{1}{\Delta_{i k} \Delta_{j k}} v_{k}, \\
b_{i j k l} & =\frac{1}{\Delta_{i j} \Delta_{i k} \Delta_{i l}} v_{i}-\frac{1}{\Delta_{i j} \Delta_{j k} \Delta_{j l}} v_{j}+\frac{1}{\Delta_{i k} \Delta_{j k} \Delta_{k l}} v_{k}-\frac{1}{\Delta_{i l} \Delta_{j l} \Delta_{k l}} v_{l} .
\end{aligned}
$$

The sign changes are due to a reordering of the indices since $\Delta_{i j}=-\Delta_{j i}$. If $v_{i}, v_{j}, v_{k}, v_{l}$ are given in the form (2.2), that is, if

$$
\left(\begin{array}{c}
v_{i} \\
v_{j} \\
v_{k} \\
v_{l}
\end{array}\right)=\left(\begin{array}{ccccccc}
1 & 0 & \cdots & \cdots & \cdots & \cdots & 0 \\
1 & \Delta_{i j} & 0 & \ddots & \cdots & \cdots & \vdots \\
1 & \Delta_{i k} & \Delta_{i k} \Delta_{j k} & \ddots & \cdots & \cdots & \vdots \\
1 & \Delta_{i l} & \Delta_{i l} \Delta_{j l} & \Delta_{i l} \Delta_{j l} \Delta_{k l} & 0 & \cdots & 0
\end{array}\right) \text {, }
$$

then $b_{i}=(1,0, \ldots, 0), b_{i j}=(0,-1,0, \ldots, 0), b_{i j k}=(0,0,1,0, \ldots, 0)$ and $b_{i j k l}=(0,0$, $0,-1,0, \ldots, 0)$. In general, $b_{1}, b_{12}, \ldots, b_{12 \cdots d+1}$ look like $(0, \ldots, 0, \pm 1,0, \ldots, 0)$ when $v_{1}, \ldots, v_{d+1}$ are of the form (2.2).

The following proposition collects the basic properties on these vectors.

\section{Proposition 2.5.}

1. For any nonempty set $S \subset[n]$, we have $b_{S} \in \mathbb{Z}^{d+1}$.

2. Let $S \subset[n]$ and $a, b \in S$ with $a \neq b$. Then we have a recursion formula

$$
b_{S}=\frac{1}{\Delta_{b a}} b_{S \backslash\{a\}}+\frac{1}{\Delta_{a b}} b_{S \backslash\{b\}} .
$$

3. For any distinct $d+1$ indices $i_{1}, \ldots, i_{d+1} \in[n]$ (not necessarily ordered), the vectors

form a $\mathbb{Z}$-basis for $\mathbb{Z}^{d+1}$.

$$
b_{i_{1}}, b_{i_{1} i_{2}}, b_{i_{1} i_{2} i_{3}}, \ldots, b_{i_{1} \cdots i_{d+1}}
$$

4. If \#S $\geq d+2$, then $b_{S}=0$. 
Proof. The second statement can be verified by elementary computations, using $\Delta_{i j}+\Delta_{j k}=\Delta_{i k}$ for $i, j, k \in[n]$.

To prove the first statement, we consider the components of $b_{S}$ as rational functions in $\tau_{i}, i \in S$. By induction on $\# S$, we prove the following statement. The components of $b_{S}$ are symmetric polynomials in $\tau_{i}, i \in S$, and their coefficients depend only on \#S.

If $\# S=1$, then $b_{S}=b_{i}=v_{i}=\left(1, \tau_{i}, \tau_{i}^{2}, \ldots, \tau_{i}^{d}\right)$, thus the claim holds. Now consider a set $S$ with at least two distinct elements $a, b$. Let

$$
f_{j}\left(\tau_{a}, \tau_{i}, i \in S\right), f_{j}\left(\tau_{b}, \tau_{i}, i \in S\right)
$$

be the $j$ th components of $b_{S \backslash b}, b_{S \backslash a}$, respectively. Then the difference between these polynomials is zero if we set $\tau_{a}=\tau_{b}$, hence the quotient

$$
\frac{f_{j}\left(\tau_{a}, \tau_{i}, i \in S\right)-f_{j}\left(\tau_{b}, \tau_{i}, i \in S\right)}{\tau_{a}-\tau_{b}}
$$

is a polynomial as claimed. It is obviously symmetric in $a$ and $b$. Since we are free to choose any two elements of $S$, it is symmetric in all variables. The coefficients of the polynomial depend only on $\# S$, so the claim is proven. Note that the degree of the polynomial decreases by one by taking the quotient. Since the degree of the components of $v_{i}$ is at most $d+1$, we conclude that $b_{S}=0$ for $\# S \geq d+2$.

To prove the third statement, we first note that the vertices $v_{i_{1}}, \ldots, v_{i_{d+1}}$ are linearly independent. Take an element $x \in \mathbb{Z}^{d+1}$ and write it as $x=\sum \lambda_{j} v_{i_{j}}$. By considering $\sigma_{\left\{i_{1}, \ldots, i_{d}\right\}}(x)$, we can say that the coefficient $\lambda_{i_{d+1}}$ is of the form

$$
\lambda_{i_{d+1}}=\frac{k}{\prod_{j=1}^{d} \Delta_{i_{j} i_{d+1}}}
$$

for an integer $k$. Thus, $x+(-1)^{d} k b_{i_{1} \ldots i_{d+1}} \in \mathbb{Z}^{d+1}$ is a vector in the subspace spanned by $v_{i_{1}}, \ldots, v_{i_{d}}$. These vectors define a $(d-1)$-dimensional cyclic polytope again, so we can proceed by induction and obtain a representation of $x$ as a $\mathbb{Z}$-linear combination of the $b_{i_{1}}, b_{i_{1} i_{2}}, \ldots, b_{i_{1} \ldots i_{d+1}}$.

We apply this construction to prove another useful fact on cyclic polytopes.

Lemma 2.6. For an integral cyclic polytope $\mathcal{P} \subset \mathbb{R}^{d}$ of dimension $d$,

$$
\mathbb{Z} \mathcal{A}_{\mathcal{P}}=\mathbb{Z}^{d+1} .
$$

Proof. First, we notice that $\mathbb{Z} \mathcal{A}_{\mathcal{P}} \subset \mathbb{Z}^{d+1}$ is obvious. To prove the reverse inclusion, we construct a basis of $\mathbb{Z}^{d+1}$ from $d+1$ points in $\mathcal{A}_{\mathcal{P}}$. We choose $d+1$ vertices $v_{1}, \ldots, v_{d+1}$ of $\mathcal{P}^{*}$ and consider the vectors

$$
b_{i_{d+1}}, b_{i_{d+1}}+b_{i_{d} i_{d+1}}, b_{i_{d+1}}+b_{i_{d} i_{d+1}}+b_{i_{d-1} i_{d} i_{d+1}}, \ldots, \sum_{l=1}^{d+1} b_{i_{l} \ldots i_{d+1}} .
$$


Let us denote them by $c_{j}:=\sum_{l=j}^{d+1} b_{i_{l} \ldots i_{d+1}}$ for $j=1, \ldots, d+1$. By Proposition 2.5(3), they constitute a $\mathbb{Z}$-basis of $\mathbb{Z}^{d+1}$. Hence, if each $c_{j}$ is contained in $\mathcal{P}^{*}$, then our claim follows. For this, let us consider the coefficient of a vertex $v_{i_{k}}$ in the sequence of

$$
b_{i_{d}}, b_{i_{d} i_{d+1}}, b_{i_{d-1} i_{d} i_{d+1}}, \ldots, b_{i_{1} \ldots i_{d+1}} .
$$

The coefficient of $v_{i_{k}}$ appears first in $b_{i_{k} \ldots i_{d+1}}$, where it has a positive sign. After that, its sign is alternating and the absolute value is nonincreasing since the denominators increase. Hence, the sum of those coefficients and thus the coefficient in $c_{j}$ is nonnegative. So, $c_{j}$ is a convex combination of the vertices of $\mathcal{P}^{*}$.

Finally, we discuss the normality of integral cyclic polytopes.

Lemma 2.7. Let $\mathcal{P}$ be an integral cyclic polytope of dimension $d$. If any simplex of dimension $d$ whose vertices are chosen from those of $\mathcal{P}$ is normal, then $\mathcal{P}$ itself is also normal.

Proof. Let $v_{1}, \ldots, v_{n}$ be the vertices of $\mathcal{P}^{*}$. The proof is by a direct application of Carathéodory's theorem (see, for example, [8, Section 7]). Let $x \in \mathbb{Z} \mathcal{A}_{\mathcal{P}} \cap \mathbb{Q}_{\geq 0} \mathcal{A}_{\mathcal{P}}$. Now, Carathéodory's theorem guarantees that there exist $d+1$ vertices $v_{i_{1}}, \ldots, v_{i_{d+1}}$ of $\mathcal{P}^{*}$ such that $x \in \mathbb{Z} \mathcal{A}_{Q} \cap \mathbb{Q}_{\geq 0} \mathcal{A}_{Q}$, where $Q=\operatorname{conv}\left(\left\{v_{i_{1}}, \ldots, v_{i_{d+1}}\right\}\right)$. Here we use the fact that $\mathbb{Z}_{\mathcal{P}}=\mathbb{Z}^{d+1}=\mathbb{Z} \mathcal{A}_{Q}$ by Lemma 2.6. If $Q$ is normal, then we have $x \in \mathbb{Z}_{\geq 0} \mathcal{A}_{Q}$, in particular, $x \in \mathbb{Z}_{\geq 0} \mathcal{A l}_{\mathcal{P}}$. This implies that $\mathcal{P}$ is normal.

\section{Normal cyclic polytopes}

Our goal in this section is to prove the following theorem.

Theorem 3.1. With the same notation as in Section 2 , if $\Delta_{i, i+1} \geq d^{2}-1$ for $1 \leq i \leq n-1$, then $\mathcal{P}=C_{d}\left(\tau_{1}, \ldots, \tau_{n}\right)$ is normal. In particular, $\gamma_{d} \leq d^{2}-1$.

Most of this section is devoted to proving the simplex case. In fact, once we know that $\mathcal{P}$ is always normal when $n=d+1$ and $\Delta_{i, i+1} \geq d^{2}-1$ for $1 \leq i \leq d$, Theorem 3.1 follows immediately from Lemma 2.7 .

Before giving a proof, we state and prove two lemmas. For the first of these, we begin by proving the following proposition.

Proposition 3.2. Let $\left(r_{1}, r_{2}, \ldots, r_{d+1}\right) \in \mathbb{Q}^{d+1}$ satisfying

$$
0 \leq r_{1} \leq r_{2} \leq \cdots \leq r_{d+1} \leq 1 \quad \text { and } \quad \sum_{i=1}^{d+1} r_{i}=m
$$

Then

(a) $\sum_{i=1}^{j} r_{i} \leq \frac{j m}{d+1}$,

(b) $\sum_{i=1}^{j} r_{d+2-i} \geq \frac{j m}{d+1}$,

for any integer $j$ with $1 \leq j \leq d+1$. 
Proof. The proof is by induction on $j$.

First, we show that $r_{1} \leq m /(d+1)$. Suppose that $r_{1}>m /(d+1)$. Then $r_{i}>$ $m /(d+1)$ for all $1 \leq i \leq d+1$ by $r_{1} \leq r_{2} \leq \cdots \leq r_{d+1}$. Thus, $m=\sum_{i=1}^{d+1} r_{i}>(d+1)$. $m /(d+1)=m$, a contradiction. Similarly, we also have $r_{d+1} \geq m /(d+1)$.

We now assume that assertions (a) and (b) hold for any integer $j^{\prime}$ with $1 \leq j^{\prime}<j$, where $j$ is some integer with $2 \leq j \leq d+1$. Let $d+1=k j+q$, where $k$ is a positive integer and $0 \leq q \leq j-1$, that is, $k$ (respectively, $q$ ) is a quotient (respectively, a remainder) of $d+1$ divided by $j$. Suppose that $\sum_{i=1}^{j} r_{i}>j m /(d+1)$. Then

$$
\sum_{i=1}^{j} r_{(k-1) j+i} \geq \sum_{i=1}^{j} r_{(k-2) j+i} \geq \cdots \geq \sum_{i=1}^{j} r_{i}>\frac{j m}{d+1}
$$

Moreover, by the induction hypothesis, $\sum_{i=k j+1}^{d+1} r_{i}=\sum_{i=1}^{q} r_{d+2-i} \geq m q /(d+1)$ when $q \neq 0$. Hence,

$$
m=\sum_{i=1}^{d+1} r_{i}>k \cdot \frac{j m}{d+1}+\frac{m q}{d+1}=m \cdot \frac{k j+q}{d+1}=m
$$

a contradiction. Therefore, assertion (a) also holds for $j$. Similarly, we also have assertion (b) for $j$, as required.

LEMMA 3.3. Let $d$ be a positive integer and $\left(r_{1}, r_{2}, \ldots, r_{d+1}\right) \in \mathbb{Q}^{d+1}$ satisfying $0 \leq r_{1} \leq$ $r_{2} \leq \cdots \leq r_{d+1} \leq 1$ and such that $\sum_{i=1}^{d+1} r_{i}$ is an integer greater than 1 . Then

$$
\max _{\substack{1 \leq i_{1}<i_{2}<\cdots<i_{l} \leq d+1 \\ 2 \leq l \leq d}}\left\{\sum_{j=1}^{l} r_{i_{j}}: \sum_{j=1}^{l-1} r_{i_{j}} \leq 1\right\} \geq 1+\frac{1}{d+1} .
$$

Proof. Let $m=\sum_{i=1}^{d+1} r_{i}$. When $m>d$, we must have $r_{i}=1$ for $1 \leq i \leq d+1$ and $m=d+1$ by our assumption. Thus, we may assume that $2 \leq m \leq d$. Let $M$ denote the value of the left-hand side of (3.1).

First step. Assume that $m-1>\lfloor(d+1) / 2\rfloor$. Then, by Proposition 3.2, $r_{d}+r_{d+1} \geq$ $2 m /(d+1)$, while $r_{d} \leq 1$. Hence,

$$
M \geq r_{d}+r_{d+1} \geq \frac{2 m}{d+1}>\frac{2}{d+1}\left(\left\lfloor\frac{d+1}{2}\right\rfloor+1\right) \geq \frac{2}{d+1}\left(\frac{d}{2}+1\right)=1+\frac{1}{d+1} .
$$

Second step. Assume that $m-1 \leq\lfloor(d+1) / 2\rfloor$ and let $d+1=k m+q$, where $k$ is a positive integer and $0 \leq q \leq m-1$, that is, $k$ (respectively, $q$ ) is a quotient (respectively, a remainder) of $d+1$ divided by $m$.

If we suppose that $\sum_{j=0}^{k-1} r_{j m+q+1}>1$, then

$$
1<\sum_{j=0}^{k-1} r_{j m+q+1} \leq \sum_{j=0}^{k-1} r_{j m+q+2} \leq \cdots \leq \sum_{j=0}^{k-1} r_{j m+q+m}
$$


Thus, $m=\sum_{i=1}^{d+1} r_{i} \geq \sum_{i=q+1}^{d+1} r_{i}>m$, a contradiction. Hence,

$$
\sum_{j=0}^{k-1} r_{j m+q+1} \leq 1
$$

Third step. If we assume that $q \neq m-1$, that is, $0 \leq q \leq m-2$, then $\sum_{j=0}^{k-2} r_{j m+q+2} \leq$ $(d-q-m+1) /(d-q)$. Suppose, to the contrary, that $\sum_{j=0}^{k-2} r_{j m+q+2}>(d-q-$ $m+1) /(d-q)$. Then

$$
\frac{d-q-m+1}{d-q}<\sum_{j=0}^{k-2} r_{j m+q+2} \leq \sum_{j=0}^{k-2} r_{j m+q+3} \leq \cdots \leq \sum_{j=0}^{k-2} r_{j m+q+m+1} .
$$

Thus, $\quad \sum_{i=q+2}^{(k-1) m+q+1} r_{i}>m(d-q-m+1) /(d-q)$. Moreover, since $\sum_{i=q+2}^{d+1} r_{i}=$ $m-\sum_{i=1}^{q+1} r_{i}$, we also have $\sum_{i=(k-1) m+q+2}^{d+1} r_{i} \geq(m-1)\left(m-\sum_{i=1}^{q+1} r_{i}\right) /(d-q)$ by Proposition 3.2. Hence,

$$
\begin{aligned}
m-\sum_{i=1}^{q+1} r_{i}=\sum_{i=q+2}^{d+1} r_{i} & >\frac{m(d-q-m+1)}{d-q}+\frac{(m-1)\left(m-\sum_{i=1}^{q+1} r_{i}\right)}{d-q} \\
& =\frac{m(d-q)}{d-q}-\frac{(m-1) \sum_{i=1}^{q+1} r_{i}}{d-q} \geq m-\sum_{i=1}^{q+1} r_{i},
\end{aligned}
$$

a contradiction. Here, since $m-1 \leq\lfloor(d+1) / 2\rfloor \leq(d+1) / 2$ and $0 \leq q \leq m-2<d$, we have $m+q \leq 2 m-2 \leq d+1$, which means that $(m-1) /(d-q) \leq 1$. Thus,

$$
\sum_{j=0}^{k-2} r_{j m+q+2} \leq \frac{d-q-m+1}{d-q}
$$

Similarly, if we assume that $q=m-1$, then

$$
\sum_{j=0}^{k-1} r_{j m+1} \leq \frac{d-m+2}{d+1}
$$

Fourth step. In this step, we prove that

$$
\sum_{j=0}^{k-1} r_{j m+q+1}+r_{d+1} \geq 1+\frac{1}{d+1}
$$

We assume that $0 \leq q \leq m-2$. Suppose, to the contrary, that $\sum_{j=0}^{k-1} r_{j m+q+1}+r_{d+1}<$ $1+1 /(d+1)$. Then $\sum_{j=1}^{k-1} r_{j m+q+1}+r_{d+1}<1+1 /(d+1)-r_{q+1}<1+1 /(d-q)-r_{q+1}$. 
Thus,

$$
\begin{aligned}
1+\frac{1}{d-q}-r_{q+1} & >\sum_{j=1}^{k-1} r_{j m+q+1}+r_{k m+q} \geq \sum_{j=1}^{k-1} r_{j m+q}+r_{k m+q-1} \geq \cdots \\
& \geq \sum_{j=1}^{k-1} r_{j m+q+1-(m-2)}+r_{k m+q-(m-2)}=\sum_{j=0}^{k-2} r_{j m+q+3}+r_{(k-1) m+q+2} .
\end{aligned}
$$

Moreover, by the third step, we also have $\sum_{j=0}^{k-2} r_{j m+q+2} \leq(d-q-m+1) /(d-q)$. Hence,

$$
\begin{aligned}
m-\sum_{i=1}^{q+1} r_{i}=\sum_{i=q+2}^{d+1} r_{i} & <m-1+\frac{m-1}{d-q}-(m-1) r_{q+1}+\frac{d-q-m+1}{d-q} \\
& =m-(m-1) r_{q+1} \leq m-(q+1) r_{q+1} \leq m-\sum_{i=1}^{q+1} r_{i}
\end{aligned}
$$

a contradiction. Similarly, when $q=m-1$, if we suppose that $\sum_{j=1}^{k} r_{j m}+r_{k m+m-1}<$ $1+1 /(d+1)$, then

$$
1+\frac{1}{d+1}>\sum_{j=1}^{k} r_{j m}+r_{k m+m-1} \geq \sum_{j=1}^{k} r_{j m-1}+r_{k m+m-2} \geq \cdots \geq \sum_{j=0}^{k-1} r_{j m+2}+r_{k m+1}
$$

and $\sum_{j=0}^{k-1} r_{j m+1} \leq(d-m+2) /(d+1)$ by the third step, so we obtain $m=\sum_{i=1}^{d+1} r_{i}<$ $m-1+(m-1) /(d+1)+(d-m+2) /(d+1)=m$, a contradiction.

Fifth step. Thanks to the second and fourth steps,

$$
M \geq \sum_{j=0}^{k-1} r_{j m+q+1}+r_{d+1} \geq 1+\frac{1}{d+1}
$$

as desired.

LeMma 3.4. Let $l$ be an integer with $l \geq 2$ and $i_{1}, \ldots, i_{l}$ distinct integers. We set

$$
Z_{l}(j)=\frac{\prod_{k=1}^{j-1} \Delta_{i_{k} i_{j}}}{\prod_{1 \leq k \leq l, k \neq j}\left|\Delta_{i_{k} i_{j}}\right|} p_{j}+\frac{\prod_{k=1}^{j-1} \Delta_{i_{k} i_{j+1}}}{\prod_{1 \leq k \leq l, k \neq j+1}\left|\Delta_{i_{k} i_{j+1}}\right|} p_{j+1}+\cdots+\frac{\prod_{k=1}^{j-1} \Delta_{i_{k} i_{l}}}{\prod_{1 \leq k \leq l, k \neq l}\left|\Delta_{i_{k} i_{l}}\right|} p_{l}
$$

for $2 \leq j \leq l$. Then, for any $2 \leq j \leq l-1$,

$$
\begin{gathered}
Z_{l}(j)=\frac{\prod_{k=1}^{j-1} \Delta_{i_{k} i_{j}}}{\prod_{1 \leq k \leq l, k \neq j}\left|\Delta_{i_{k} i_{j}}\right|} p_{j}+\frac{1}{\Delta_{i_{j} i_{j+1}}} Z_{l}(j+1)-\frac{1}{\Delta_{i_{j} i_{j+1}} \Delta_{i_{j} i_{j+2}}} Z_{l}(j+2) \\
+\cdots+(-1)^{l-j+1} \frac{1}{\prod_{k=j+1}^{l} \Delta_{i_{j} i_{k}}} Z_{l}(l) .
\end{gathered}
$$


A proof is given by elementary computations.

Now, Lemma 3.4 says that if $Z_{l}(j+1), \ldots, Z_{l}(l)$ are integers, then there exists an integer $p_{j}$ such that $Z_{l}(j)$ becomes an integer. In fact, since

$$
\frac{1}{\Delta_{i_{j} i_{j+1}}} Z_{l}(j+1)-\cdots+(-1)^{l-j+1} \frac{1}{\prod_{k=j+1}^{l} \Delta_{i_{j} i_{k}}} Z_{l}(l)=\frac{P}{C},
$$

where $P$ is some integer and $C=\prod_{k=j+1}^{l}\left|\Delta_{i_{j} i_{k}}\right|$, and the numerator (respectively, the denominator) of $\prod_{k=1}^{j-1} \Delta_{i_{k} i_{j}} / \prod_{1 \leq k \leq l, k \neq j}\left|\Delta_{i_{k} i_{j}}\right|$ is either 1 or -1 (respectively, $C$ ), it is obvious that there exists an integer $p_{j}$ such that $Z_{l}(j)$ becomes an integer.

Let $Q \subset \mathbb{R}^{N}$ be an integral convex polytope of dimension $d$. In general, when $\mathbb{Z} \mathcal{A}_{Q}=\mathbb{Z}^{N+1}$, in order to prove that $Q$ is normal, it suffices to show that for any $\alpha=\left(m, \alpha_{1}, \ldots, \alpha_{N}\right) \in \mathbb{Z}_{\mathcal{A}_{Q}} \cap \mathbb{Q}_{\geq 0} \mathcal{A}_{Q}=\mathbb{Q}_{\geq 0} \mathcal{A}_{Q} \cap \mathbb{Z}^{N+1}$ with $m \geq 2$, we find $\alpha^{\prime} \in Q^{*} \cap$ $\mathbb{Z}^{N+1}$ and $\alpha^{\prime \prime} \in \mathbb{Q}_{\geq 0} \mathcal{A}_{Q} \cap \mathbb{Z}^{N+1}$ with $\alpha=\alpha^{\prime}+\alpha^{\prime \prime}$. (This is equivalent to proving that $Q$ satisfies the integer decomposition property.) In particular, when $Q$ is a simplex, since there exists a unique $\left(r_{1}, \ldots, r_{d+1}\right) \in \mathbb{Q}^{d+1}$ such that $\alpha=\sum_{i=1}^{d+1} r_{i} u_{i}$ and $\sum_{i=1}^{d+1} r_{i}=$ $m$, where $u_{1}, \ldots, u_{d+1}$ are the vertices of $Q^{*}$, we may find $\left(r_{1}^{\prime}, \ldots, r_{d+1}^{\prime}\right) \in \mathbb{Q}^{d+1}$ with $\sum_{i=1}^{d+1} r_{i}^{\prime} u_{i} \in Q^{*} \cap \mathbb{Z}^{N+1}$ and $\left(r_{1}^{\prime \prime}, \ldots, r_{d+1}^{\prime \prime}\right) \in \mathbb{Q}^{d+1}$ with $\sum_{i=1}^{d+1} r_{i}^{\prime \prime} u_{i} \in \mathbb{Q}_{\geq 0} \mathcal{A}_{Q} \cap \mathbb{Z}^{N+1}$ satisfying $r_{i}^{\prime}+r_{i}^{\prime \prime}=r_{i}$ for $1 \leq i \leq d+1$.

Hence, it is enough to show that for any $\alpha=\sum_{i=1}^{d+1} r_{i} u_{i} \in \mathbb{Q}_{\geq 0} \mathcal{A}_{Q} \cap \mathbb{Z}^{N+1}$ with $\sum_{i=1}^{d+1} r_{i} \geq 2$, there exists $\left(r_{1}^{\prime}, \ldots, r_{d+1}^{\prime}\right) \in \mathbb{Q}^{d+1}$ such that

$$
\sum_{i=1}^{d+1} r_{i}^{\prime}=1, \quad 0 \leq r_{i}^{\prime} \leq r_{i} \text { for } 1 \leq i \leq d+1 \quad \text { and } \quad \sum_{i=1}^{d+1} r_{i}^{\prime} u_{i} \in \mathbb{Z}^{N+1} .
$$

We are now in a position to verify the normality of integral cyclic polytopes in the case where $n=d+1$ and $\Delta_{i, i+1} \geq d^{2}-1$ for $1 \leq i \leq d$. Let $\mathcal{P}$ be such a cyclic polytope. Let $m \geq 2$ be an integer and $\alpha$ an element in $\mathbb{Z}_{\mathcal{P}} \cap \mathbb{Q}_{\geq 0} \mathcal{A}_{\mathcal{P}}=\mathbb{Q}_{\geq 0} \mathcal{A}_{\mathcal{P}} \cap \mathbb{Z}^{d+1}$ with first coordinate $m$. Since $\mathcal{P}^{*}$ is a simplex of dimension $d$, there exists a unique $\left(r_{1}, \ldots, r_{d+1}\right) \in \mathbb{Q}^{d+1}$, where $\sum_{i=1}^{d+1} r_{i}=m$, such that $\alpha=\sum_{i=1}^{d+1} r_{i} v_{i}$. Then what we must do is to show that there exists $\left(r_{1}^{\prime}, \ldots, r_{d+1}^{\prime}\right) \in \mathbb{Q}^{d+1}$ such that

$$
\sum_{i=1}^{d+1} r_{i}^{\prime}=1, \quad 0 \leq r_{i}^{\prime} \leq r_{i} \text { for } 1 \leq i \leq d+1 \quad \text { and } \quad \sum_{i=1}^{d+1} r_{i}^{\prime} v_{i} \in \mathbb{Z}^{d+1}
$$

First step. If there exists $r_{i}$ with $r_{i} \geq 1$, say, $r_{1}$, then we may set $r_{1}^{\prime}=1$ and $r_{2}^{\prime}=\cdots=r_{d+1}^{\prime}=0$. Moreover, when $m \geq d+1$, since $\sum_{i=1}^{d+1} r_{i}=m$ and $r_{i} \geq 0$, there is at least one $r_{i}$ with $r_{i} \geq 1$. Thus, we may assume that

$$
2 \leq m \leq d \quad \text { and } \quad 0 \leq r_{i} \leq 1 \quad \text { for } 1 \leq i \leq d+1 .
$$

Second step. By Lemma 3.3, there exist $r_{i_{1}}, \ldots, r_{i_{l}}$ among $\left(r_{1}, \ldots, r_{d+1}\right)$ such that $\sum_{j=1}^{l} r_{i_{j}} \geq 1+1 /(d+1)$ and $\sum_{j=1}^{l-1} r_{i_{j}} \leq 1$, where $0 \leq r_{i_{1}} \leq \cdots \leq r_{i_{l}} \leq 1$ and $2 \leq l \leq d$, 
although we do not know whether $1 \leq i_{1}<\cdots<i_{l} \leq d+1$. However, we assume that $0 \leq r_{i_{l}} \leq r_{i_{l-1}} \leq \cdots \leq r_{i_{1}} \leq 1$, that is,

$$
\sum_{j=2}^{l} r_{i_{j}} \leq 1 \quad \text { and } \quad \sum_{j=1}^{l} r_{i_{j}} \geq 1+\frac{1}{d+1} .
$$

Let $D=d^{2}-1$. Thus, $\left|\Delta_{i j}\right| \geq D$ for any $1 \leq i \neq j \leq d+1$. We now set $\epsilon(l)=$ $(l-1) / D$ for $2 \leq l \leq d$. Then it is easy to see that $\epsilon(l)$ enjoys the following properties:

$$
\begin{gathered}
\epsilon(l) \geq \sum_{a=2}^{l} \frac{1}{D^{a-1}}, \quad \frac{1}{d+1}=\epsilon(d)>\epsilon(d-1)>\cdots>\epsilon(2), \\
\epsilon(l)-\frac{l-j+1}{D^{j-1}}>\epsilon(j-1) \quad \text { for } 3 \leq j \leq l .
\end{gathered}
$$

In the following two steps, by induction on $l$, we prove that if $\sum_{j=1}^{l} r_{i_{j}} \geq 1+\epsilon(l)$ and $\sum_{j=2}^{l} r_{i_{j}} \leq 1$, then there exists $\left(r_{1}^{\prime}, \ldots, r_{d+1}^{\prime}\right) \in \mathbb{Q}^{d+1}$ satisfying (3.2). Once we know this, we obtain the required assertion from $2 \leq l \leq d$ and $1 /(d+1)=\epsilon(d) \geq \epsilon(l)$.

Third step. Assume that $l=2$, that is, $r_{i_{1}}+r_{i_{2}} \geq 1+1 / D$, where $0 \leq r_{i_{2}} \leq r_{i_{1}} \leq 1$.

Let $p$ be a nonnegative integer satisfying

$$
\frac{p}{\left|\Delta_{i_{1} i_{2}}\right|} \leq r_{i_{2}}<\frac{p+1}{\left|\Delta_{i_{1} i_{2}}\right|}
$$

Then it is clear that there exists such a unique nonnegative integer $p$. Let $r_{i_{2}}^{\prime}=$ $p /\left|\Delta_{i_{1} i_{2}}\right|, r_{i_{1}}^{\prime}=1-r_{i_{2}}^{\prime}$ and $r_{j}^{\prime}=0$ for any $j$ with $j \in[d+1] \backslash\left\{i_{1}, i_{2}\right\}$. Thus, $\sum_{i=1}^{d+1} r_{i}^{\prime}=1$ and $0 \leq r_{i_{2}}^{\prime} \leq r_{i_{2}}$. Moreover, since $r_{i_{2}} \leq 1$, we have $r_{i_{1}}^{\prime}=1-r_{i_{2}}^{\prime} \geq 1-r_{i_{2}} \geq 0$. In addition, by $r_{i_{1}}+r_{i_{2}} \geq 1+1 / D$ and $\left|\Delta_{i_{1} i_{2}}\right| \geq D$, we also have

$$
r_{i_{1}}-r_{i_{1}}^{\prime}=r_{i_{1}}-1+\frac{p}{\left|\Delta_{i_{1} i_{2}}\right|} \geq \frac{1}{D}-r_{i_{2}}+\frac{p}{\left|\Delta_{i_{1} i_{2}}\right|} \geq \frac{p+1}{\left|\Delta_{i_{1} i_{2}}\right|}-r_{i_{2}}>0 .
$$

On the other hand, by Proposition 2.2, we may consider $v_{i_{1}}$ and $v_{i_{2}}$ as $v_{i_{1}}=$ $(1,0, \ldots, 0)$ and $v_{i_{2}}=\left(1, \Delta_{i_{1} i_{2}}, 0, \ldots, 0\right)$. Obviously, $\sum_{i=1}^{d+1} r_{i}^{\prime} v_{i} \in \mathbb{Z}^{d+1}$.

Fourth step. Assume that $l \geq 3$. For each $j$ with $2 \leq j \leq l$, we define each nonnegative integer $p_{j}$ as follows. Let $p_{l}$ be a nonnegative integer which satisfies

$$
\frac{p_{l}}{\prod_{k=1}^{l-1}\left|\Delta_{i_{k} i_{l}}\right|} \leq r_{i_{l}}<\frac{p_{l}+1}{\prod_{k=1}^{l-1}\left|\Delta_{i_{k} i_{l}}\right|},
$$

and for $2 \leq j \leq l-1$, let $p_{j}$ be an integer which satisfies $Z_{l}(j) \in \mathbb{Z}$ and

$$
\frac{p_{j}}{\prod_{1 \leq k \leq l, k \neq j}\left|\Delta_{i_{k} i_{j}}\right|} \leq r_{i_{j}}<\frac{p_{j}+\prod_{k=j+1}^{l}\left|\Delta_{i_{j} i_{k}}\right|}{\prod_{1 \leq k \leq l, k \neq j}\left|\Delta_{i_{k} i_{j}}\right|}
$$


where $Z_{l}(j)$ is as in Lemma 3.4. Thanks to Lemma 3.4, if $Z_{l}(j+1), \ldots, Z_{l}(l) \in \mathbb{Z}$, then there exists an integer $p_{j}$ with $Z_{l}(j) \in \mathbb{Z}$ and each $p_{j}$ is uniquely determined by the above inequalities. Remark that we do not know whether $p_{j}$ is nonnegative except for $p_{l}$. However, in our case, we may assume that $p_{2}, \ldots, p_{l-1}$ are all nonnegative because of the following discussions. Suppose, to the contrary, that there is $j^{\prime}$ with $p_{j^{\prime}}<0$. Let $q_{j^{\prime}} \in \mathbb{Z}_{\geq 0}$ be a minimal nonnegative integer satisfying

$$
\begin{aligned}
& \frac{\prod_{k=1}^{j^{\prime}-1} \Delta_{i k} i_{j^{\prime}}}{\prod_{1 \leq k \leq l, k \neq j^{\prime}}\left|\Delta_{i_{k} j_{j^{\prime}}}\right|} q_{j^{\prime}}+\frac{1}{\Delta_{i_{j^{\prime}} j_{j^{\prime}+1}}} Z_{l}\left(j^{\prime}+1\right)-\frac{1}{\Delta_{i_{j^{\prime}} i_{j^{\prime}+1}} \Delta_{i_{j^{\prime}} i_{j^{\prime}+2}}} Z_{l}\left(j^{\prime}+2\right) \\
& \quad+\cdots+(-1)^{l-j^{\prime}+1} \frac{1}{\prod_{k=j^{\prime}+1}^{l} \Delta_{i^{\prime} i^{\prime}}} Z_{l}(l) \in \mathbb{Z} .
\end{aligned}
$$

In particular, it follows from the minimality of $q_{j^{\prime}}$ that $0 \leq q_{j^{\prime}}<\prod_{k=j^{\prime}+1}^{l}\left|\Delta_{i_{j^{\prime}} i_{k}}\right|$. By our assumption, $q_{j^{\prime}} / \prod_{1 \leq k \leq l, k \neq j^{\prime}}\left|\Delta_{i_{k} i_{j^{\prime}}}\right|>r_{i_{j^{\prime}}}$. Thus,

$$
r_{i_{l}} \leq \cdots \leq r_{i^{\prime}}<\frac{q_{j^{\prime}}}{\prod_{1 \leq k \leq l, k \neq j^{\prime}}\left|\Delta_{i_{j^{\prime}} i_{k}}\right|}<\frac{\prod_{k=j^{\prime}+1}^{l}\left|\Delta_{i^{\prime} i_{k}}\right|}{\prod_{1 \leq k \leq l, k \neq j^{\prime}}\left|\Delta_{i_{k} i_{j^{\prime}}}\right|}=\frac{1}{\prod_{k=1}^{j^{\prime}-1}\left|\Delta_{i_{k} i_{j^{\prime}}}\right|} \leq \frac{1}{D^{j^{\prime}-1}},
$$

so $\sum_{j=j^{\prime}}^{l} r_{i_{j}}<\left(l-j^{\prime}+1\right) / D^{j^{\prime}-1}$. From $\sum_{j=1}^{l} r_{i_{j}} \geq 1+\epsilon(l)$ and (3.3),

$$
\sum_{j=1}^{j^{\prime}-1} r_{i_{j}}>1+\epsilon(l)-\frac{l-j^{\prime}+1}{D^{j^{\prime}-1}}>1+\epsilon\left(j^{\prime}-1\right)
$$

when $j^{\prime} \geq 3$. Hence, we may skip this case by the induction hypothesis. When $j^{\prime}=2$, we have $r_{i_{1}}>1+\epsilon(l)-(l-1) / D=1$, a contradiction.

By using the above $p_{j}$, we define $r_{1}^{\prime}, \ldots, r_{d+1}^{\prime}$ by setting

$$
r_{a}^{\prime}= \begin{cases}\frac{p_{j}}{\prod_{1 \leq k \leq l, k \neq j}\left|\Delta_{i_{k} i_{j} \mid}\right|} & \text { if } a=i_{j} \in\left\{i_{2}, \ldots, i_{l}\right\}, \\ 1-\sum_{j=2}^{l} r_{i_{j}}^{\prime} & \text { if } a=i_{1}, \\ 0 & \text { otherwise. }\end{cases}
$$

In particular, $\sum_{a=1}^{d+1} r_{a}^{\prime}=1$. By definition of $r_{i_{2}}^{\prime}, \ldots, r_{i_{l}}^{\prime}$, we have $0 \leq r_{i_{j}}^{\prime} \leq r_{i_{j}}$ for $2 \leq j \leq l$. Moreover, from $\sum_{j=2}^{l} r_{i_{j}} \leq 1$, we also have $r_{i_{1}}^{\prime}=1-\sum_{j=2}^{l} r_{i_{j}}^{\prime} \geq 1-\sum_{j=2}^{l} r_{i_{j}} \geq 0$. In addition, from $\sum_{j=1}^{l} r_{i_{j}} \geq 1+\epsilon(l)$ and (3.3), we also have

$$
\begin{aligned}
r_{i_{1}}-r_{i_{1}}^{\prime} & =r_{i_{1}}-1+\sum_{j=2}^{l} \frac{p_{j}}{\prod_{1 \leq k \leq l, k \neq j}\left|\Delta_{i_{k} i_{j}}\right|} \geq \epsilon(l)-\sum_{j=2}^{l} r_{i_{j}}+\sum_{j=2}^{l} \frac{p_{j}}{\prod_{1 \leq k \leq l, k \neq j}\left|\Delta_{i_{k} i_{j}}\right|} \\
& \geq \sum_{j=2}^{l}\left(\frac{p_{j}}{\prod_{1 \leq k \leq l, k \neq j}\left|\Delta_{i_{k} i_{j}}\right|}+\frac{1}{D^{j-1}}-r_{i_{j}}\right) \geq \sum_{j=2}^{l}\left(\frac{p_{j}+\prod_{k=j+1}^{l}\left|\Delta_{i_{j} i_{k}}\right|}{\prod_{1 \leq k \leq l, k \neq j}\left|\Delta_{i_{k} i_{j}}\right|}-r_{i_{j}}\right) \\
& >0 .
\end{aligned}
$$


Finally, we verify that $\sum_{i=1}^{d+1} r_{i}^{\prime} v_{i} \in \mathbb{Z}^{d+1}$. Again, by Proposition 2.2, we may consider $v_{i_{1}}, \ldots, v_{i_{l}}$ as follows:

$$
\left(\begin{array}{c}
v_{i_{1}} \\
v_{i_{2}} \\
\vdots \\
v_{i_{l}}
\end{array}\right)=\left(\begin{array}{cccccccc}
1 & 0 & \cdots & \cdots & \cdots & 0 & \cdots & 0 \\
1 & \Delta_{i_{1} i_{2}} & 0 & \ddots & \ddots & \vdots & & \vdots \\
1 & \Delta_{i_{1} i_{3}} & \Delta_{i_{1} i_{3}} \Delta_{i_{2} i_{3}} & \ddots & \ddots & \vdots & & \vdots \\
\vdots & \vdots & \vdots & \ddots & \ddots & \vdots & & \vdots \\
1 & \Delta_{i_{1} i_{l}} & \Delta_{i_{1} i_{l}} \Delta_{i_{2} i_{l}} & \cdots & \prod_{k=1}^{l-1} \Delta_{i_{k} i_{l}} & 0 & \cdots & 0
\end{array}\right) .
$$

Hence, it is easy to check that

$$
\sum_{i=1}^{d+1} r_{i}^{\prime} v_{i}=\sum_{j=1}^{l} r_{i_{j}}^{\prime} v_{i_{j}}=\left(1, Z_{l}(2), Z_{l}(3), \ldots, Z_{l}(l), 0, \ldots, 0\right) \in \mathbb{Z}^{d+1}
$$

proving the assertion.

Remark 3.5. Since each lattice length of an edge $\operatorname{conv}\left(\left\{v_{i}, v_{j}\right\}\right)$ of $\mathcal{P}^{*}$ coincides with $\Delta_{i j}$, where $i<j$, it follows immediately from [3, Theorem 1.3(b)] that $\mathcal{P}$ is normal if $\Delta_{i, i+1} \geq d(d+1)$ for $1 \leq i \leq n-1$. (We are grateful to Gábor Hegedüs for drawing [3, Theorem 1.3(b)] to our attention.) Thus, our constraint $\Delta_{i, i+1} \geq d^{2}-1$ on integral cyclic polytopes is better than the general case, but this bound is still very rough. For example, $C_{3}(0,1,2,3)$ is normal, while $\Delta_{12}=\Delta_{23}=\Delta_{34}=1<8$. Similarly, $C_{4}(0,1,3,5,6)$ is also normal, although $\Delta_{12}=\Delta_{45}=1$ and $\Delta_{23}=\Delta_{34}=2$.

\section{Cyclic polytopes that are not very ample}

Our goal in this section is to prove the following theorem.

THEOREM 4.1. Let $d$ and $n$ be positive integers satisfying $n \geq d+1$ and $d \geq 4$. If $\Delta_{12}=1$ or $\Delta_{n-2, n-1}=1$, then $C_{d}\left(\tau_{1}, \ldots, \tau_{n}\right)$ is not very ample.

We obtain Theorem 4.1 as a conclusion of Proposition 4.2 and Corollary 4.3 below.

Proposition 4.2. Let $\mathcal{P}=C_{4}\left(\tau_{1}, \ldots, \tau_{n}\right)$. If $\Delta_{23}=1$ or $\Delta_{n-2, n-1}=1$, then $\mathcal{P}$ is not very ample.

Proof. Thanks to Lemma 2.3, by symmetry, we assume that $\Delta_{23}=1$. Consider the set

$$
\mathcal{A}_{\mathcal{P}, 3}:=\left\{x-v_{3}: x \in \mathcal{P}^{*} \cap \mathbb{Z}^{5}\right\}
$$

We will prove that the monoid $\mathbb{Z}_{\geq 0} \mathcal{A}_{\mathcal{P}, 3}$ is not normal, thus there exists a vector $p \in \mathbb{Z}_{\mathcal{P}, 3} \cap \mathbb{Q}_{\geq 0} \mathcal{A}_{\mathcal{P}, 3}=\mathbb{Q}_{\geq 0} \mathcal{A}_{\mathcal{P}, 3} \cap \mathbb{Z}^{5}$ such that $p \notin \mathbb{Z}_{\geq 0} \mathcal{A}_{\mathcal{P}, 3}$. Then, for every integer $k \geq 1$, we have $k v_{3}+p \in\left(\mathbb{Z}_{\mathcal{P}} \cap \mathbb{Q}_{\geq 0} \mathcal{A}_{\mathcal{P}}\right) \backslash \mathbb{Z}_{\geq 0} \mathcal{A}_{\mathcal{P}} ;$ see [1, Exercise 2.23]. Hence, $\mathcal{P}$ is not very ample. 
In the following, we denote the facet of $\mathcal{P}^{*}$ spanned by the vertices $v_{i}, v_{j}, v_{k}$ and $v_{l}$ by $\mathcal{F}_{i j k l}$. Moreover, we denote the corresponding linear form by $\sigma_{i j k l}$. Note that every facet of $\mathcal{P}^{*}$ containing $v_{3}$ also defines a facet of $\mathbb{Q}_{\geq 0} \mathcal{A}_{\mathcal{P}, 3}$.

The following vector has the required properties:

$$
\begin{aligned}
p:= & b_{23}+b_{134}+b_{12345} \\
= & \frac{\Delta_{12} \Delta_{15}+1}{\Delta_{12} \Delta_{13} \Delta_{14} \Delta_{15}} v_{1}+\frac{1}{\Delta_{23}}\left(1-\frac{1}{\Delta_{12} \Delta_{24} \Delta_{25}}\right) v_{2}-\frac{1}{\Delta_{23}}\left(1+\frac{\Delta_{23} \Delta_{35}-1}{\Delta_{13} \Delta_{34} \Delta_{35}}\right) v_{3} \\
& \quad+\frac{\Delta_{24} \Delta_{45}-1}{\Delta_{14} \Delta_{24} \Delta_{34} \Delta_{45}} v_{4}+\frac{1}{\Delta_{15} \Delta_{25} \Delta_{35} \Delta_{45}} v_{5} .
\end{aligned}
$$

First, we have $p \in \mathbb{Z}^{5}$ from Proposition 2.5(1). Then, by the second representation of $p$, it is a positive linear combination of the vectors $v_{1}-v_{3}, v_{2}-v_{3}, v_{4}-v_{3}$ and $v_{5}-v_{3}$. Thus, $p \in \mathbb{Q}_{\geq 0} \mathcal{A}_{\mathcal{P}, 3}$. Moreover, since we assume that $\Delta_{23}=1$, the coefficient of $v_{3}$ is less than -1 . Hence, $p$ lies beyond the facet $\mathcal{F}_{1245}$ which is a facet of $\mathcal{P}^{*}$ by Gale's evenness condition (Proposition 2.1). Thus, $p \notin \mathcal{A}_{\mathcal{P}, 3}$.

It remains to show that $p$ cannot be written as a sum $\sum w_{j}$ with $w_{j} \in \mathcal{A}_{\mathcal{P}_{3,3}}$. Suppose that we have such a representation. Then we remark that $p$ has at least two summands. Consider a facet $\mathcal{F}_{1234}$. Then $\sigma_{1234}(p)=1 /\left(\Delta_{15} \Delta_{25} \Delta_{35} \Delta_{45}\right) \sigma_{1234}\left(v_{5}\right)=1$. Since $\sigma_{1234}\left(w_{j}\right) \geq 0, \sigma_{1234}\left(w_{j}\right)=0$ for every summand $w_{j}$ except one. Choose one $w_{j} \neq 0$ with $\sigma_{1234}\left(w_{j}\right)=0$ and denote it by $w$. Further, we set $w^{\prime}:=p-w \in \mathbb{Z}_{\geq 0} \mathcal{A}_{\mathcal{P}, 3}$ the remaining sum. By Carathéodory's theorem, there exist vertices $v_{i_{1}}, \ldots, v_{i_{4}}$ of $\mathcal{P}^{*}$ and nonnegative numbers $\lambda_{j} \geq 0$ such that $w^{\prime}=\sum_{j=1}^{4} \lambda_{j}\left(v_{i_{j}}-v_{3}\right)$. Let $i_{4}$ be the greatest of those indices. Since $\sigma_{1234}\left(w^{\prime}\right)=1$ and $\sigma_{1234}\left(v_{i_{4}}\right)=\Delta_{1 i_{4}} \Delta_{2 i_{4}} \Delta_{3 i_{4}} \Delta_{4 i_{4}}$, we conclude that

$$
\lambda_{4} \leq \frac{1}{\Delta_{1 i_{4}} \Delta_{2 i_{4}} \Delta_{3 i_{4}} \Delta_{4 i_{4}}} .
$$

But the vertices $v_{i_{1}}, \ldots, v_{i_{4}}$ and $v_{3}$ define an integral cyclic polytope, thus the denominator of the coefficient of $v_{i_{4}}$ has to be a divisor of $\Delta_{i_{1} i_{4}} \Delta_{i_{2} i_{4}} \Delta_{i_{3} i_{4}} \Delta_{3 i_{4}}$. This is only possible if $\left\{i_{1}, i_{2}, i_{3}\right\}=\{1,2,4\}$. Thus, $w^{\prime}$ lies in the cone generated by $v_{1}-v_{3}, v_{2}-v_{3}, v_{4}-v_{3}$ and $v_{i_{4}}-v_{3}$. Note that $\sigma_{1234}(w)=0$ implies that $w$ lies in the cone generated by $v_{1}-v_{3}, v_{2}-v_{3}$ and $v_{4}-v_{3}$. Thus we can replace the polytope $\mathcal{P}^{*}$ by the polytope $Q^{*}$ whose vertices are $v_{1}, \ldots, v_{5}$ and $v_{i_{4}}$. The reason for doing this is that we know the facets of $Q^{*}$. Here, $i_{4}=5$ is possible.

We consider the representation

$$
w=a_{1} b_{3}+a_{2} b_{23}+a_{3} b_{123}+a_{4} b_{1234}
$$

with integer coefficients $a_{1}, a_{2}, a_{3}, a_{4}$. This is possible from Proposition 2.5(3). Since $w$ is in the cone generated by $v_{1}-v_{3}, v_{2}-v_{3}$ and $v_{4}-v_{3}$, we have $a_{1}=0$. Now consider a facet $\mathcal{F}_{123 i_{4}}$ of $Q^{*}$. We compute

$$
\sigma_{123 i_{4}}(p)=\frac{1}{\Delta_{45}}\left(\Delta_{24} \Delta_{45}-1\right) \Delta_{4 i_{4}}+\frac{1}{\Delta_{45}} \Delta_{5 i_{4}}=\Delta_{24} \Delta_{4 i_{4}}-1 .
$$


Moreover, $\sigma_{123 i_{4}}(w)=-a_{4} \Delta_{4 i_{4}}$. From $0 \leq \sigma_{123 i_{4}}(w) \leq \sigma_{123 i_{4}}(p)$, we conclude that $0 \leq$ $-a_{4} \leq \Delta_{24}-1$. Here we used the fact that $a_{4}$ is an integer. Next, consider a facet $\mathcal{F}_{2345}$. We compute $\sigma_{2345}(w)=a_{3} \Delta_{14} \Delta_{15}+a_{4} \Delta_{15}$ and $\sigma_{2345}(p)=\Delta_{12} \Delta_{15}+1$. As before, we conclude that $0 \leq a_{3} \Delta_{14}+a_{4} \leq \Delta_{12}$. However, these two constraints can only be satisfied by $a_{3}=a_{4}=0$, because $\Delta_{14}=\Delta_{12}+\Delta_{24}$ and $\Delta_{15}>1$. Finally, we consider a facet $\mathcal{F}_{134 i_{4}}$. By computing $\sigma_{134 i_{4}}(w)=a_{2} \Delta_{12} \Delta_{24} \Delta_{2 i_{4}}$ and $\sigma_{134 i_{4}}(p)=\Delta_{12} \Delta_{24} \Delta_{2 i_{4}}-1$, we conclude that $a_{2}=0$. But this means that $w=0$, in contradiction to $w \neq 0$.

Using this proposition, we also obtain the following corollary.

Corollary 4.3. Let $\mathcal{P}=C_{d}\left(\tau_{1}, \ldots, \tau_{n}\right)$, where $d \geq 5$. If there is some $i$ with $2 \leq i \leq$ $n-2$ such that $\Delta_{i, i+1}=1$, then $\mathcal{P}$ is not very ample.

Proof. We prove this by induction on $d$.

When $d=5$, let $\mathcal{F}_{i}=\operatorname{conv}\left(\left\{v_{1}, v_{i}, v_{i+1}, v_{i+2}, v_{i+3}\right\}\right)$ for $2 \leq i \leq n-3$ and $\mathcal{F}_{n-2}=$ $\operatorname{conv}\left(\left\{v_{n-4}, v_{n-3}, v_{n-2}, v_{n-1}, v_{n}\right\}\right)$. By Gale's evenness condition, each $\mathcal{F}_{i}$ is a facet of $\mathcal{P}^{*}$. When $\Delta_{i, i+1}=1$ for some $i$ with $2 \leq i \leq n-2$, it then follows from Proposition 4.2 that $\mathcal{F}_{i}$ is not very ample. Thus, $\mathcal{P}$ itself is not very ample either. (See [7, Lemma 1].)

Now let $d \geq 6$. For $2 \leq i \leq n-d+2$, we set

$$
\mathcal{F}_{i}= \begin{cases}\operatorname{conv}\left(\left\{v_{1}, v_{i}, \ldots, v_{i+d-2}\right\}\right) & \text { when } d \text { is odd } \\ \operatorname{conv}\left(\left\{v_{i-1}, v_{i}, \ldots, v_{i+d-2}\right\}\right) & \text { when } d \text { is even }\end{cases}
$$

Again, Gale's evenness condition guarantees that each $\mathcal{F}_{i}$ is a facet of $\mathcal{P}^{*}$. When $\Delta_{i, i+1}=1$ for some $i$ with $2 \leq i \leq n-2$, since each facet is also an integral cyclic polytope of dimension $d-1$, either $\mathcal{F}_{i}$ or $\mathcal{F}_{d-n+2}$ is not very ample by the induction hypothesis. Therefore, $\mathcal{P}$ is not very ample.

In the case where $d=2$, it is well known that there exists a unimodular triangulation for every integral convex polytope of dimension two. Therefore, integral convex polytopes of dimension two are always normal.

In the case where $d=3$, exhaustive computational experience leads us to give the following conjecture.

Conjecture 4.4. All cyclic polytopes of dimension three are normal.

Moreover, by computational experience together with Proposition 4.2, we also conjecture a complete characterization of normal cyclic polytopes of dimension four.

Conjecture 4.5. A cyclic polytope of dimension four is normal if and only if

$$
\Delta_{23} \geq 2 \quad \text { and } \quad \Delta_{n-2, n-1} \geq 2 .
$$

By considering the foregoing two conjectures and Theorem 3.1, the following statement seems natural to us.

Conjecture 4.6. If $\mathcal{P}=C_{d}\left(\tau_{1}, \ldots, \tau_{n}\right)$ is normal and $\mathcal{P}^{\prime}=C_{d}\left(\tau_{1}^{\prime}, \ldots, \tau_{n}^{\prime}\right)$ satisfies $\tau_{j}^{\prime}-\tau_{i}^{\prime} \geq \Delta_{i j}$ for all $1 \leq i<j \leq n$, then $\mathcal{P}^{\prime}$ is also normal. 
Finally, we also state the following conjecture.

Conjecture 4.7. If an integral cyclic polytope is very ample, then it is also normal.

Actually, it often happens that a very ample integral convex polytope is also normal, that is to say, the normality of an integral convex polytope is equivalent to what it is very ample. Hence, the above conjecture occurs naturally. On the other hand, it is also known that there exists an integral convex polytope which is not normal but very ample. See [1, Exercise 2.24].

\section{References}

[1] W. Bruns and J. Gubeladze, Polytopes, Rings and K-theory (Springer, Heidelberg, 2009).

[2] B. Grünbaum, Convex Polytopes, 2nd edn (Springer, Heidelberg, 2003).

[3] J. Gubeladze, 'Convex normality of rational polytopes with long edges', Adv. Math. 230 (2012), 372-389.

[4] T. Hibi, Algebraic Combinatorics on Convex Polytopes (Carslaw Publications, Glebe, NSW, Australia, 1992).

[5] T. Hibi, A Higashitani, L. Katthän and R. Okazaki, Toric rings arising from cyclic polytopes. arXiv: $1204.5565 \mathrm{v} 1$.

[6] H. Ohsugi and T. Hibi, 'Normal polytopes arising from finite graphs', J. Algebra 207 (1998), 409-426.

[7] H. Ohsugi and T. Hibi, 'Nonvery ample configurations arising from contingency tables', Ann. Inst. Statist. Math. 62 (2010), 639-644.

[8] A. Schrijver, Theory of Linear and Integer Programming (John Wiley \& Sons, Chichester, 1986).

[9] R. P. Stanley, Combinatorics and Commutative Algebra, 2nd edn (Birkhäuser, Boston, 1995).

[10] G. M. Ziegler, Lectures on Polytopes (Springer, Heidelberg, 1995).

TAKAYUKI HIBI, Department of Pure and Applied Mathematics, Graduate School of Information Science and Technology,

Osaka University, Toyonaka, Osaka 560-0043, Japan

e-mail: hibi@math.sci.osaka-u.ac.jp

AKIHIRO HIGASHITANI, Department of Pure and Applied Mathematics, Graduate School of Information Science and Technology,

Osaka University, Toyonaka, Osaka 560-0043, Japan

e-mail: a-higashitani@cr.math.sci.osaka-u.ac.jp

LUKAS KATTHÄN, Fachbereich Mathematik und Informatik, Philipps-Universität Marburg, 35032 Marburg, Germany

e-mail: katthaen@mathematik.uni-marburg.de

RYOTA OKAZAKI, Faculty of Education, Fukuoka University of Education, Munakata, Fukuoka 811-4192, Japan

e-mail: rokazaki@fukuoka-edu.ac.jp 